\title{
Éléphants vedettes de théâtre: Mlle. Djeck ou la starification animale dans la première moitié du XIXe siècle
}

\section{Theatre's Elephant Vedettes: Mlle. Djeck or the Animal Starification in the first half of Nineteenth-Century}

\author{
Ma Teresa Lajoinie Domínguez \\ Universidad de Valencia \\ maria.t.lajoinie@uv.es
}

\section{Resumen}

El objetivo del presente artículo es determinar la existencia de vedettes animales rastreando, para ello, la superposición de aquellos mecanismos y dinámicas conducentes a la adquisición de dicho estatus en el caso de actores y actrices humanos. Cogiendo como ejemplo paradigmático el caso de la elefanta Mlle. Djeck, presente en los escenarios europeos del siglo XIX, se analizarán los elementos que permiten tanto la inclusión del animal-actor en el fenómeno de la celebridad, cuanto aquellas características que resultan en su singularización. Asimismo, se estudiarán las nociones de voyerismo e intimidad como principales ejes en torno a los cuales se construye la nueva relación entre público y vedette propia de esta figura. Por último, se atenderá al papel fundamental de la prensa, así como otros medios mediáticos, en cuanto que intermediarios necesarios para la construcción, promoción y popularización de la vedette y/o celebridad.

\section{Palabras clave}

elefante, vedette-animal, celebridad, teatro, zooescenografía.

\begin{abstract}
The main of this article is to determine the existence of the animal stars by analysing the mechanisms and dynamics conducive to the acquisition of this status in the case of human actors and actresses. Taking as a paradigmatic example the case of the elephant Mlle. Djeck, present in the European stages of the 19th century, will analyse the elements that allow both the inclusion of the animal-actor in the phenomenon of celebrity, as well as those characteristics that result in its singularization. Likewise, the notions of voyeurism and intimacy will be studied as the main axes around which the new relationship between the public and the star of this figure is built. Lastly, the fundamental role of the press, as well as other media, will be addressed as intermediaries necessary for the construction, promotion and popularization of the star and/or celebrity.
\end{abstract}

\section{Key words}

elephant, animal-vedette, celebrity, theatre, zoo scenography. 


\section{Introduction}

Le 10 juillet 1837, le journal Le Charivari offre à ses lecteurs un article qui pourrait être qualifié de rétrospective aux touches nécrologiques, revendication d'une actrice qui avait marqué le panorama dramatique, tant sur le plan national qu'international. Le journaliste A. S., signataire de Quelques fleurs sur une tombe -tel est le titre de son écrit- regrette et reproche la perte que la scène européenne vient de souffrir avec la mort de Mlle. Djeck et, comme si d'un Zola avant la lettre il s'agissait, accuse ouvertement et explicitement la Suisse:

[...] De commettre un forfait exécrable. Cette chaste et paisible contrée, que nous représentions si vertueuses avec ses grands lacs et ses petits châlets [sic], s'est à jamais deshonorée [sic] par une exécution dont le souvenir fera pâlir encore long-temps [sic] les nations effrayées. Adieu donc, Suisse corrompue, que Guillaume-Tell renierait avec indignation; je te fuis plein d'horreur; car tu as terni tes neiges si fières jadis de leur blancheur immaculée! Que la malédiction céleste s'appesantisse sur toi! Puisse M. Alexandre Dumas te décrire encore, dans de nouvelles Impressions de voyage! et puisse M. Hyppolyte Souverain répandre à cent mille exemplaires sa Suisse Pittoresque!

L'exécution de celle que la presse étrangère avait nommée "[the] elephantine 'star"” (Actors by Daylight, 1838: 255), (re)intensifie l'intérêt pour cette actrice colossale, reprenant ici un des adjectifs les plus couramment utilisés tout au long d'une trajectoire qui s'étend durant trois décennies. Ce panégyrique met en lumière que Mlle. Djeck était loin d'être un simple pachyderme présenté dans les salles théâtrales du Paris dix-neuviémiste et devient la preuve de la célébrité dont elle jouissait. Mlle. Djeck, ou miss Djeck, fait partie des nombreux acteurs quadrupèdes qui inondent la scène boulevardière parisienne au XIX ${ }^{\mathrm{e}}$ siècle. Éléphants, mais aussi chiens, oiseaux, tigres ou lions, intègrent nombre de troupes se produisant à l'époque et deviennent les protagonistes de spectacles zooscénographiques qui attirent le public de la première moitié du siècle. La popularité de ces représentations résulte, en effet, d'un contexte historique déterminé qui est favorable à la (omni)présence de l'animal scénique. Le projet colonial, les nouvelles théories sur les origines animales de l'humanité ou les changements progressifs du statut de l'animal non-humain (allant du concept d'objet à celui d'être sensible ${ }^{1}$ ) qui le font intégrer la famille humaine, figurent parmi les principaux points qui expliquent le recours itératif aux acteurs de quatre pattes.

1 Les transformations s'opèrent de manière continue mais certainement lente, les modifications étant de nos jours encore en train de s'accomplir. Bien que le siècle des Lumières voit un engouement pour les animaux, conséquence des courants de sensibilité ayant pour chefs de file Rousseau, Voltaire ou Condillac, c'est après la Révolution que le débat s'étend et quitte l'exclusivité des milieux cultivés. Au XIX ${ }^{\mathrm{e}}$ la première loi visant la protection des animaux, la loi Grammont (1850), est approuvée et surgissent les premières sociétés protectrices (la Royal Society for the Prevention of Cruelty to Animals en 1824 à Londres, la SPA en 1845 à Paris). Cependant, comme Fanny Dupas (2005: 11) le signale, l'animal continue d'être considéré juridiquement un objet - meuble par nature ou immeuble par destination - propriété de l'homme (voir la loi rurale du 28 septembre -6 octobre 1791, les articles 522 et 528 du code civil de 1804, puis les articles 452 - 455 du code pénal de 1810). Il faudra attendre plus d'un siècle pour qu'en 1963 la France introduise dans sa législation l'acte cruel contre 
Notons, par ailleurs, qu'au XIX ${ }^{\mathrm{e}}$ siècle, les arts du spectacle sont en pleine mutation. La généralisation des loisirs dans la société bourgeoise de l'époque fait du théâtre un divertissement consommé par une grande partie de la population française, et notamment parisienne. Paris est alors la capitale des spectacles, le boulevard du Temple le centre névralgique d'une vie mondaine où, comme le souligne Geneviève Faye (2010), les différentes classes sociales se réunissent, parfois dans une même salle. S'encanaillant ou voulant échapper aux difficultés que la réalité impose, aristocratie, bourgeoisie et classes populaires font des soirées de théâtre le délassement privilégié de cette société qui aime voir, mais aussi être vue. L'hétérogénéité est ainsi une marque caractérisant un public qui, même avec la restructuration impériale ordonnée par les décrets de 1806 et 1807, ne cesse de se rendre aux établissements théâtraux. De même, le genre boulevardier se heurte aux limites dérivant du système de privilèges qui ne sera réellement aboli qu'en 1864. Son affiche étant limitée à des genres qualifiés de mineurs, en accord avec un circuit dramatique lui aussi désigné comme secondaire, les directeurs et artistes doivent faire preuve d'ingéniosité pour remplir le parterre. Le théâtre cheminant vers sa conversion définitive en industrie, les bénéfices économiques se dévoilent comme l'une des préoccupations majeures et situent subséquemment le public au centre d'un processus qui culmine dans la mise en scène. Ainsi, les spectateurs s'érigent en vrai moteur de création artistique ce qui permet aux salles boulevardières de combler leurs atteintes, leurs goûts déterminant, donc, en première instance, la composition de l'affiche, voire la production et/ou écriture des œuvres. Cette dynamique est également applicable aux artistes (qu'ils soient bipèdes ou quadrupèdes), car ils sont aussi soumis aux préférences des spectateurs. Le public, compris dans le sens habermassien du terme, agit alors en sorte de critique dramatique, cette opinion publique devenant l'un des principaux éléments sanctionnateurs. La carrière des acteurs, tout comme le succès des pièces théâtrales, est liée, non seulement à leur qualité - dans la déclamation, dans le jeu ou dans la mise en scène -, mais aussi à la faveur d'un public qui assiste en foule voir son artiste privilégié. Ceci met en relief l'importance capitale de la question de la célébrité, et souligne, par conséquent, l'apparition d'un système de vedettariat au sein des arts de la scène au XIX ${ }^{\mathrm{e}}$ siècle.

Or, la célébrité en tant que phénomène a été abordée par de nombreuses études qui, dans un premier temps, l'ont principalement connectée avec le surgissement du star-system, faisant de cette manifestation un événement prenant place au $\mathrm{XX}^{\mathrm{e}}$ siècle. Les Stars, volume qu'Edgar Morin publie aux éditions du Seuil en 1957, est devenu un classique dans les travaux

\footnotetext{
l'animal comme délit. Sa catégorisation juridique en tant qu'être sensible n'arrivera qu'en 1976. Ainsi, le code rural, dans son article L214-1, affirme que "tout animal étant un être sensible doit être placé par son propriétaire dans des conditions compatibles avec les impératifs biologiques de son espèce". Cette catégorisation sera également reconnue en 2015 par le code civil, qui dans son Livre II article 515-14 définit les animaux comme "des êtres vivants doués de sensibilité". Malgré cette avancée, il existe encore une certaine distorsion entre la catégorie d'être sensible qui leur est octroyée et "le régime des biens" (code civil, Livre II, art. 515-14) auquel ils continuent d'être actuellement soumis. Pour plus d'information sur les progrès en matière de protection animale en France consulter: Canselier, Sonia (2017).
} 
qui ont visé la définition et caractérisation de la figure de la star. Cette dernière aurait fondamentalement prospéré dans le milieu cinématographique, puis dans une société de masses triomphante, car pleinement consolidée. Une des principales caractéristiques de ces figures publiques relevée par Edgar Morin (1957) est la mythification, voire divinisation, dont elles font l'objet. Cependant, la présente approche s'inscrit dans la lignée d'études plus récentes qui défendent que la naissance de ce mouvement de reconnaissance est antérieure à la création du cinéma. C'est donc au sein du domaine dramatique qu'il faut chercher l'apparition du concept de "vedette", intimement lié à l'idée de célébrité, et de là, d'un vedettariat qui engendrerait, après modifications et adaptations, la notion "d'étoile" (star) cultivée par le septième art, puis popularisée par l'industrie hollywoodienne. Ainsi, certains spécialistes font remonter bien avant le $\mathrm{XX}^{\mathrm{e}}$ siècle l'apparition du vedettariat dont l'éclosion se situerait entre le XVII ${ }^{e}$ et $\mathrm{XIX}^{\mathrm{e}}$ siècle (Filippi, Harvey \& Marchand, 2017), et plus concrètement à partir du XVIII ${ }^{\mathrm{e}}$ siècle (Tillyard, 2005; Jones \& Joule, 2018). Dans cette ligne se situe Antoine Lilti (2014) qui défend que la célébrité, et par conséquent les premières vedettes, voient le jour dès la seconde moitié du siècle des Lumières, à la suite notamment de la configuration d'une "société du spectacle". Le XVIII' siècle aurait ainsi entrepris la modification des rapports entre public et artistes qui les conduisent à se constituer en figures publiques. En ce sens, Talma et Mlle. Mars sont maintes fois requis en exemples d'une notoriété acquise avant l'avènement du XIX ${ }^{\mathrm{e}}$ siècle. En effet, tel que souligné par Mary Luckhurst et Jane Moody (2005), cette célébrité est possible grâce à l'essor de la presse, ainsi que d'autres moyens de médiatisation, car ils deviennent un mécanisme essentiel dans la construction, promotion et diffusion de l'image publique des vedettes. Bien que la date de parution d'un système conduisant à la notoriété publique varie en fonction des auteurs, ce qui reste indéniable est que, dans la période dix-neuviémiste, le phénomène du vedettariat est une réalité.

Voyant le succès incontestable de Mlle. Djeck, ainsi que les passions qu'elle suscite chez le journaliste qui recense son exécution en 1837, la question qui se pose dans cet article est de savoir si, sur le modèle déjà mis en place pour les artistes humains, serait-il possible de dégager l'existence parallèle de vedettes animales. Il s'agit, en outre, de déterminer les mécanismes qui ont permis à Mlle. Djeck de parvenir à la notoriété publique et de mettre simultanément en lumière les analogies, mais aussi les possibles particularités, entre les dynamiques conduisant à la popularisation de l'acteur animal et humain. Pour ce faire, l'analyse portera, dans un premier temps, sur les éléments qui caractérisent et différencient la vedette des autres artistes qui, comme elle, sont engagés dans le circuit spectaculaire. Ceci nous permettra de considérer le statut particulier de l'animal célèbre lui permettant de participer complètement du phénomène de la célébrité, dans les termes conçus pour les acteurs humains. L'étude se penchera alors sur les notions d'intimité et de voyeurisme, en tant que concepts clés venant définir les nouveaux rapports entre public et vedette. Il sera aussi question de souligner les particularités qui relèvent du statut de célébrité animale et qui divergent des approches à la figure de la vedette humaine. Finalement, le rôle de la presse en tant qu'intermédiaire indis- 
Anales de Filología Francesa, n. ${ }^{\circ}$ 29, 2021

$\mathrm{M}^{\mathrm{a}}$ TERESA LAJOINIE DOMÍNGUEZ

pensable dans la construction de l'image publique de la vedette, puis sa valeur promotionnelle et publicitaire, seront abordés.

\section{Entre le 'It'? de l'animalité et l'analogie de l'humanisation: à la conquête de la popularité}

Mlle. Djeck ${ }^{3}$ débarque en Europe en 1806 provenant de l'Inde. Pendant cette première période, elle connaît plusieurs propriétaires et intègre différentes ménageries, passant inaperçue pour le public, tout comme pour la presse de l'époque. Il faut attendre deux décennies pour que, le 4 juillet 1829, ait lieu son début théâtral à l'établissement parisien du Cirque Olympique qui l'engage pour remplir le rôle d'éléphant sacré dans le drame à grand spectacle intitulé L'Éléphant du roi de Siam. Dès le lendemain, les journalistes qui s'attardent à faire les premières critiques de la pièce relèvent, non pas tellement la valeur littéraire de la même -certains parlant d'une trame faible ${ }^{4}$, mais l'habileté avec laquelle Djeck réalise chacun des numéros qui lui sont assignés. D'ailleurs, Le Messagers des chambres du 6 juillet ne fait nulle mention aux acteurs humains dans un article entièrement consacré à la figure de l'animal, encore anonyme (et toujours mâle) pour le grand public, laissant entrevoir que le succès du spectacle repose essentiellement sur sa performance, voire sa seule présence sur scène:

On aurait désiré que l'éléphant seul se chargeât de la pantomime, des péripéties, du dénouement et du dialogue de la pièce nouvelle dans laquelle $i l$ débutait; mais on n'a pu échapper à une pièce. [...] Nous ne nous opposerions nullement au bonheur de Nadir et d'Imadora, si les trois entre'actes [sic] nécessaires ne duraient pas une heure chacun. Cet inconvénient, joint au peu de commodité des loges du Cirque-Olympique, pourrait nuire au succès mérité de cette nouveauté dramatique et zoologique. [...] à la fin de la représentation, il [l'éléphant, donc Mlle. Djeck] a été redemandé, et $i l$ est venu, sans cornac, recevoir avec dignité les applaudissements du public ${ }^{5}$.

Loin d'être une exception, la prépondérance de l'actrice pachyderme dans ces écrits s'avère une constante, comme le prouve également l'exemple du Journal des comédiens du 5 juillet 1829 qui tient principalement à faire l'éloge de "l'éléphant, dont on parlait depuis si

2 Joseph Roach (2005) donne le nom de "It" à toutes les caractéristiques possédées par une personne et qui la dotent d'un magnétisme, d'un charisme, qui résulte dans la capacité d'attraction sur les autres. Les éléments constitutifs de ce "It" sont multiples et variés, J. Roach (2005: 16) affirmant son caractère polymorphe.

3 Les graphies du nom varient en fonction des sources consultées. Ainsi, il est possible de retrouver non seulement les formes sus-citées, donc Mlle. ou miss Djeck, mais aussi autres écritures comme "D'jeck" (Fennell, 1841: 397; Goldsmith, 1842: 303) et "Madame ou Mademoiselle Jack” (Brown, 1834: 380; Hughes, 1883: 382). Cette dernière, semble être plus courante dans le contexte anglophone, ainsi que germanique, Djeck (et ses variantes) étant en revanche plus usitées dans les milieux français et/ou francophones.

4 Voir le numéro 343 du Messager des chambres du mercredi 9 décembre 1829, dans lequel le journaliste décrit "le nœud de cette composition [comme étant] extrêmement faible, mais la mise en scène est [en revanche] de toute beauté".

5 Les italiques ont été introduits par nous-même dans le but de faire remarquer au lecteur qu'effectivement, tel qu'il a été auparavant signalé, pendant les débuts de sa carrière théâtrale, Mlle. Djeck fut considérée un éléphant mâle. 
long-temps (sic)". Cependant, l'ascension de Mlle. Djeck se trouve encore à une phase initiale, l'intérêt qu'on lui porte se limitant à ses hautes capacités interprétatives qui permettent toutefois de prévoir une fructueuse carrière artistique. Il est intéressant de souligner le fait que, comme la citation permet de le confirmer, la présence de l'éléphant(e) était attendue par le public, évoquant l'existence préalable d'une certaine publicité à son égard. En tant qu'instance de promotion -autant économique que sociale- la publicité est un des éléments clés, non seulement dans le système industriel dans lequel se trouvait alors immergé le théâtre, mais aussi dans le phénomène de la célébrité. Remarquée du public et mise en vedette par la critique théâtrale, un des principaux "opérateurs de sélection-consécration" (Dubois, 2001) conduisant à la reconnaissance de la part des pairs, il est possible de conclure que l'actrice était prise au milieu de mécanismes relevant du mouvement de la célébrité. Notons qu'au sein de cette société du spectacle, et notamment dans le cas des salles secondaires, ainsi que du système de vedettariat, la faveur du public se constitue en moyen venant déterminer l'acquisition de cette nouvelle forme de reconnaissance sociale hautement médiatisée (Filippi, Harvey \& Marchand, 2017). De leur côté, les articles journalistiques qui font la critique du spectacle agissent en agents publicitaires et médiatiques, élargissant, à leur tour, le nombre potentiel de spectateurs et participant, de ce fait, du succès de la pièce théâtrale, de l'artiste, mais surtout, de la renommée que cette dernière acquiert progressivement. Soirée après soirée, les applaudissements recueillis par Mlle. Djeck, constamment redemandée à la fin de chaque représentation, montrent la curiosité qu'elle suscite chez les spectateurs,

Yet notwithstanding two such very formidable obstacle to agility [her height and her weight], she performs the whole of her part, even to her final acknowledgments to the audience, upon being called for, à la Français, after the curtain has fallen, with an ease and elegance which very many of her biped colleagues would do well to imitate. (Edinburgh Literary Journal, 1829: 403)

L'actrice éléphantine se confirme donc en tant que principale réclame, autant de la pièce que de l'établissement, tel que Michel Morin le soutient lorsqu'il affirme que les incalculables succès de miss Djeck firent reporter au Cirque Olympique cent mille écus (1833: 315). En effet, l'attention dont Djeck est protagoniste relève d'un rapport avec le public qui est symptomatique du passage progressif du statut de "simple" actrice, qu'elle occupait avant son arrivée à la scène parisienne, à celui d'actrice populaire. Cette popularité repose notamment dans l'idée de singularité venant caractériser toute vedette de théâtre.

Quelles raisons sont donc aux origines de cette singularisation faisant de l'éléphant(e) la vraie héroïne et de l'ouvrage dramatique et des écrits journalistiques, contrairement à des acteurs humains devenus secondaires et systématiquement négligés par les quotidien? En premier lieu, il faut considérer que, traditionnellement conçu en tant que revers, voire opposé de l'humanité, l'animal(-ité) émerge encore à cette époque comme l'altérité par excellence qui permet de marquer la divergence physique, mais aussi comportementale. Ce binôme, qui 
puise ses racines dans ce que Philippe Descola (2005) a nommé le "grand partage", fait que, vis-à-vis de la norme que l'humain incarne, l'idée de singularité prenne corps dans l'animal. En conséquence, puisque représentante de ce règne du naturel, Mlle. Djeck se situe "hors du commun, au-delà du quotidien", et devient, per se, cette "quelque chose à noter" que toute célébrité doit posséder (Blewitt, 2013: 327). C'est précisément cette idée qui transparaît dans l'article que Théophile Gautier consacre au spectacle de Les Éléphants de la pagode (1845), quand il soutient que,

\begin{abstract}
Chaque fois qu'on annonce un éléphant, un rhinocéros, un hippopotame, un crocodile, un serpent boa plus ou moins instruit, nous y courons, toute affaire cessante. On a si peu d'occasions, dans la vie civilisée, de voir les ouvrages du bon Dieu! -Quelles énormités monstrueuses, quels étranges caprices s'est permis le souverain sculpteur, surtout dans les premiers jours de la création! Alors, il ne craignait pas de manquer de matière, il modelait largement et sans économiser la terre glaise: c'était le temps des mammouths [...] De cette nature colossale, il est resté quelques témoins, les moins disproportionnés avec le monde actuel. Leur grandeur relative et l'étrangeté de leurs formes les faits regarder avec curiosité et stupeur; [...] Ces pensées nous préoccupaient l'autre soir, au Cirque [Olympique], beaucoup plus que la pièce, exactement taillé sur le patron de celle qui a servi autrefois pour Kiouni ou mademoiselle Djeck. (1859: 171-172)
\end{abstract}

Comme l'écrivain parnassien le laisse entrevoir, les spectacles zooscénographiques du boulevard du Temple deviennent l'occasion pour la population française de la première moitié du siècle de confronter "an animal otherness powerfully antithetical to human" (Mi1ler, 2012: 37), et par extension, de voir et de jouir de l'inouï et du différent. En effet, dans le cas concret de Mlle. Djeck, cette singularité s'accroît du fait que, dans L'Éléphant du roi de Siam, elle est le seul individu-animal sur scène, par opposition à une humanité, qui trop commune au public pour être profondément surpris, se multiplie dans plus d'une dizaine d'acteurs. Par ailleurs, la rareté de l'éléphant dans le contexte européen, puisqu'espèce exogène, s'unit au caractère spectaculaire de sa taille. Avant Jumbo, pour qui une grande partie de sa popularité reposa aussi sur ce trait physique 7 , Djeck fut "le plus grand éléphant que

6 Dans Par-delà nature et culture (2005), Philippe Descola évoque le paradigme dichotomique qui oppose nature et culture, la première réalité excluant automatiquement la seconde, car traditionnellement considérées en tant que notions contraires. Or, ces deux concepts sont à connecter avec le pair animal-humain qui participe de cette pensée binaire à la consolidation de laquelle il contribue. En ce sens, le XIX ${ }^{\mathrm{e}}$ siècle entend l'idée de civilisation comme synonyme de culture, leur proposant comme antonymes les notions de sauvagerie et de nature respectivement. De ce fait, puisque "la culture relève du propre de l'humain (démarqu[ant] le civilisé du sauvage)" (Gouabault \& Burton-Jeangros, 2010: 301), par effet d'analogie, la nature devient le propre de l'animal, et ce notamment dans le cas de l'animal sauvage. Ainsi, l'animal non-humain émerge désormais en tant que principal symbole synecdotique pour évoquer ladite notion de nature.

7 Le recours à cette caractéristique comme réclame visant à encourager la promotion d'animaux en figures publiques, puis en célébrités, se consolide avec le temps au point de devenir une stratégie mise en place de manière itérative. Djeck et Jumbo au XIX ${ }^{\mathrm{e}}$ siècle, mais aussi le gorille Guy, exhibé au zoologique de Londres entre les décennies de 1960 et 1970 jusqu'à sa mort en 1974, ont été fréquemment décrits avec des adjectifs cherchant à souligner une corporalité imposante qui les rend singuliers (Blewitt, 2013). Néanmoins, l'anatomie divergente n'est pas, en elle-même, le seul élément déterminant la célébrité des individus-animaux plus haut mentionnés, 
l'on ait vu en Europe et en Amérique" (Anonyme, 1832: 5). Cette "nature colossale" qui fait du pachyderme une "énormité monstrueuse" (Gautier, 1859: 171-172), intensifie par conséquent l'écart entre le public et une artiste qui, de ce fait, devient unique.

Toutefois, comme John Blewitt (2013) ou Antoine Lilti (2014) l'ont mis en lumière, la vedette n'est pas seulement caractérisée par l'idée de particularité, car c'est dans l'équilibre entre le commun et/ou ordinaire et l'entièrement extraordinaire que cette figure se situe. À la fois singulière et semblable aux autres, la personne célèbre s'avère essentiellement ambivalente. Bien qu'apparemment paradoxal, ce mécanisme où similitude et dissimilitude se combinent est caractéristique du système de vedettariat, que ce dernier atteigne un individu humain ou animal. À cet effet, les exercices réalisés par Mlle. Djeck permettent de la rapprocher des acteurs humains, et par extension des spectateurs eux-mêmes, par le biais d'une performance qui, en écho avec Antonia Losano (2017), dénaturalise le comportement animal. Sur ce point, la scène 14 (quatrième partie, acte II), correspondant au grand banquet royal, se confirme comme un des numéros les plus représentatifs, ainsi que l'un des plus acclamés de la pièce ${ }^{8}$. Affublée d'une serviette en cachemire et disposant d'une énorme sonnette pour appeler les esclaves chargés du service de table, Djeck mange, débouche plusieurs bouteilles qu'elle boit ultérieurement et s'essuie elle-même la trompe. Ainsi, la performance fait de l'éléphant un imitateur des actions humaines. Participer des codes culturels qui ritualisent un acte de subsistance comme est le fait de s'alimenter conduit le pachyderme à agir de manière analogue à l'humain, promouvant, en dernière instance, leur identification mutuelle.

Cependant, le parallélisme s'effectue, non seulement par rapport au versant performatif, mais aussi sur le plan discursif. Tel est le cas lorsque l'acteur humain est traité, comme dans The Edinburgh Literary Journal (1829), de "biped colleague" ou encore quand Mlle. Djeck est reconnue comme "artiste" (Le Mercure, 1829: 96) ou "the actor" (Calcutta Magazine, 1830). C'est donc aussi par le biais du discours que l'éléphant(e) accède au statut d'actrice, puis de célébrité (Giles, 2013) et, de là, se rapproche de l'humain. À la manière des animaux de compagnie, ces vedettes quadrupèdes deviennent, à la suite d'une identité essentiellement hybride, "humanized animals" (Danahay, 2007: 102). Ce caractère liminaire intrinsèque à l'animal-acteur est celui qui permet sa pleine intégration dans les mécanismes et stratégies liés au phénomène de la célébrité, puis d'étudier ce dernier dès une perspective s'éloignant de l'approche anthropocentrique traditionnelle. Le vedettariat semble ainsi émerger en tant qu'élément de brouillage ontologique, situation qui se confirme non seulement par le biais de l'humanisation de l'animal, mais également par l'animalisation de la vedette humaine. Or, les comparaisons entre célébrités et animaux sont aussi récurrentes. En témoigne

mais elle vient s'ajouter à d'autres traits ou situations, étant la conjonction ou l'ensemble de tous ces aspects ce qui rend possible leur constitution en tant que figures célèbres.

8 La scène est d'ailleurs reprise dans des spectacles ultérieurs, parmi lesquels Les Éléphants de la pagode de 1845, arrivant même à se constituer en un numéro détaché qui sera intercalé, comme entracte, au milieu d'autres représentations. 
le cas de Voltaire (Burney, 1773: 56) ou de Sarah Siddons, notamment à la suite de leur (sur) exhibition et, de ce fait, de leur réduction à objets pour être vus et consommés. Soulignons, finalement, que l'anthropomorphisation de la vedette éléphantine, également soutenue par une trame présentant un pachyderme doté de responsabilités institutionnelles ou de sentiments comme l'affection -ceci étant considéré comme caractéristique de l'espèce humaine- est en même temps la voie par laquelle ces acteurs "à quatre pattes" se singularisent et se différencient de leurs congénères qui, en état sauvage, maintiennent leur conduite naturelle.

\section{Du voyeurisme et autres formes d'intimité}

Visible au moment de la représentation, la scène émerge en tant qu'espace par excellence pour frôler physiquement l'artiste. En effet, "[the] theatrical space is the key crucible through which the private gets publicised and celebrity culture gets forged" (Jones \& Joule, 2018: 6). La place capitale des planches dans la construction du phénomène de la célébrité est d'autant plus évidente que, dans le cas de Djeck, ce n'est que lorsqu'elle rejoint le circuit dramatique parisien qu'elle devient réellement populaire et connue pour le public. Néanmoins, la sphère théâtrale ne satisfait qu'une partie de la curiosité que la persona (ou dans ce cas "animalia") de l'acteur suscite chez les spectateurs:

It was Saturday night; and, according to the custom, the young lady set out on her journey at midnight, day travelling being, very properly, as much avoided as possible. A great number of persona had waited outside the theatre, and, their curiosity being not quite sated, accompanied Mademoiselle to the turnpike, upon arriving at which she was left alone with her keepers. It was a beautiful night. (Actors by Daylight, 1838: 255)

Cette nécessité de voir le pachyderme en dehors de l'espace théâtral, un désir parfois qualifié de "voyeurisme" (Lilti, 2014), est à connecter avec la volonté du public de créer des liens intimes qui dépassent le contexte de la performance. Autrement dit, il s'agit de pouvoir participer de l'intimité de l'acteur ou actrice mettant en vue, sous la lumière publique, ce qui reste caché pour les spectateurs, à savoir, tout ce qui configure son existence parathéâtrale. En ce sens, les journaux et autres formes d'écriture ou de reproduction de son image, voire l'exhibition même de sa corporalité, permettent d'entrer dans sa quotidienneté et/ou de rester à proximité de Mlle. Djeck alors que la représentation est conclue et que, physiquement et visiblement, elle n'est plus accessible pour le public.

Les mois qui suivent la première représentation de L'Éléphant du roi de Siam permettent de constater la multiplication de références et écrits sur Djeck, puis les changements dans le traitement auprès de sa figure. D'un côté, elle abandonne l'anonymat auquel elle avait été soumise dans un premier temps, son sexe d'éléphant(e) femelle étant désormais connu de tous. D'un autre côté, les écrits lui étant consacrés se diversifient et cessent de parler uniquement de son savoir-faire sur la scène, et, en paroles du Messagers des chambres du 
9 décembre 1829, du "succès colossal" qu'elle retrouve à chaque établissement où elle se produit. On assiste alors à un effondrement des limites entre les sphères du public et du privé, la figure de l'actrice éléphantine dépassant le rôle incarné et s'affirmant désormais elle-même comme personnage. Ce n'est donc plus l'éléphant de Siam qui intéresse l'opinion publique, mais Mlle. Djeck.

La bravoure, le scandale, parfois même le macabre, sont ainsi exploités par les quotidiens qui décrivent, çà et là, son apparence physique, ses possibles aventures amoureuses avec un éléphant inconnu avec qui elle aurait engendrer Miss Betzy ${ }^{9}$ (Karr, 1835: 167), le spectacle que sa seule arrivée suppose ou sa prétendue mort dans un naufrage alors qu'elle revenait de sa tournée à travers les États-Unis ${ }^{10}$. La variété d'événements narrés constitue donc un caléidoscope permettant de reconstruire la biographie de Mlle. Djeck de son propre vivant, dans le même temps que le public accède à des informations, sinon des rumeurs, qui font partie de la vie privée de l'artiste. En effet, la contemporanéité entre le personnage, le public et les faits narrés sur sa persona-animalia est un trait caractéristique de la célébrité face à d'autres formes de reconnaissance publique qui se développent essentiellement de manière posthume, telle la gloire. Or, ces anecdotes qui relèvent plus du quotidien, voire de tout ce qui entoure cette pachyderme, que de sa vie professionnelle, rendent possible que le public satisfasse son "désir d'intimité à distance" (Lilti, 2014: 31). L'accès au privé que ces anecdotes offrent crée une illusion de familiarité avec l'actrice principalement fondée sur les idées de réel et d'authenticité. En conséquence, ces articles permettent de dépasser les limites spatiales et temporelles du théâtre, mais surtout de rompre avec l'imposture et la fictionnalité des représentations dramatiques, notions qui déterminent la vie et l'image publiques de l'artiste.

Cette recherche d'intimité s'exprime dans le cas de Mlle. Djeck d'une manière crue, voire brutale, lors de son exécution à Genève en 1837. Maintenue dans un fossé pendant deux mois, les autorités suisses se décident finalement à mettre à mort l'actrice éléphantine, jugée devenue folle et trop dangereuse pour la population. D'après certains journaux de l'époque, parmi lesquels le Journal des débats politiques et littéraires du 6 juillet 1837, les habitants apprennent la nouvelle de sa fin tragique en même temps que l'exécution a lieu. Or, la multiplicité de références et de détails fournis par les différents quotidiens suggère plutôt l'absence de publicité, ce qui viserait à contenir le nombre de curieux susceptibles de s'y rendre, et non pas tellement que la mise à mort fut réalisée dans l'intimité ou dans un espace privé, spécialement parce que l'exécution se fait par boulet de canon dans les remparts de la ville. Ceci

9 Éléphant(e) se produisant dans les théâtres boulevardiers dans les décennies de 1830 et 1840. Il semble que Mlle. Djeck et Miss Betzy ou Betzi (selon la source consultée), aient partagé pendant quelque temps la scène, certainement dans le spectacle de Les Deux Éléphants (1832).

10 Le Corsaire du mardi 18 janvier 1831, dans l'article Mort d'une jeune actrice, annonce que Mlle. Djeck: "Elle n'est plus!... Hélas! Blâsée [sic] par l'encens européen, elle a voulu visiter les plages américaines... La traversée lui devint funeste. De barbares matelots, menacés d'un naufrage, la précipitèrent dans l'Océan, sans pitié pour son âge et son sexe!... Pauvre Mlle Djeck! Perle des éléphans [sic], que la mer te soit légère!’. L'information est postérieurement démentie dans le journal Le Figaro du 5 février 1832 qui annonce la réapparition de l'actrice à l'établissement du Cirque Olympique. 
coïnciderait avec l'affirmation de Kurt Koenigsberger pour qui la rareté et le caractère spectaculaire, parce qu'étrange, que revêt la mort d'un éléphant, fait de ces épisodes des "events that occasioned enthusiastic and repeated narration" (2007: 77) ${ }^{11}$. Cet enthousiasme narratologique, qui confirme par ailleurs le sentiment de curiosité que Djeck continue de générer et explique une partie importante de sa popularité, est cependant à mettre en opposition avec l'accueil que la décision eut parmi l'opinion publique dix-neuviémiste, dont la presse agit en porte-parole. Ceci est d'autant plus évident que François Mayor, docteur en chirurgie chargé de l'exécution de Mlle. Djeck, est contraint de publier, entre autres dans Le Courrier français du 4 août 1837, un article, sorte de justification, dans lequel il explique la méthode de mise à mort qu'il a suivie et où il soutient avec véhémence avoir évité la souffrance de l'éléphant(e). La réaction est à connecter avec l'identité hybride qui anthropomorphise le pachyderme, résultat de son inclusion dans la culture de la célébrité, puis des courants de sensibilité à l'égard des animaux répandus dès le XVIII' ${ }^{\text {e }}$ siècle, ce qui l'éloigne du statut univoque d'animal. Notons également le côté macabre, voire sensationnaliste, de la nouvelle qui met en lumière les détails de la mort de Djeck et alimente, une fois de plus, l'intérêt autour de sa figure, la presse s'affirmant comme un des principaux mécanismes promotionnels. Confirmant l'affirmation de K. Koenigsberger, l'événement s'avère une nouvelle occasion pour (ré)écrire la vie et la mort de cette actrice, la notice ne paraissant dans pas moins de cinq journaux français, puis autant d'autres anglophones. Ainsi, le rapprochement virtuel de la vedette de la part du public est renouvelé et articulé, cela même après sa mort, par les moyens de médiatisation que la presse, et autres supports visuels comme les lithographies ${ }^{12}$, représentent.

Mais, au-delà des écrits publiés, deux circonstances restent remarquables, parce qu'extraordinaires. D’une part, le fait que l'éléphant(e) ait été dépiécée après son exécution, sa chaire étant vendue aux genevois en sorte de moyen de dédomager Lott, son dernier propriétaire. Selon le Journal des débats politiques et littéraires, "il y eut toute la journée grande affluence à la boucherie, où l'on débitait la viande du gigantesque quadrupède, et il s'est fait le soir et le lendemain dans Genève, une grande consommation d'éléphant". Le penchant voyeuriste relevé par Antoine Lilti (2014), qui caractérise les liens que le public entretient avec la vedette, est poussé à l'extrême se transformant dans le cas de Djeck en carnisme, voire cannibalisme. Dans la ligne de l'analyse que propose Claudine van Hensbergen de la vente aux enchères d'objets ayant appartenu à l'actrice anglaise Anne Oldfield, la boucherie aussi "could function, like the theatre, as a [new] site for the circulation and consumption of

11 L'appréciation est d'ailleurs déjà relevée par François Mayor qui, dans le journal Le Courrier français du 4 août 1837, soutient que "la mort d'un éléphant étant chose rare en Europe, doit nécessairement occuper le public; aussi la plupart des journaux ont-ils annoncé la fin du second, que dans l'espace de dix-sept ans, on a été obligé de tuer à Genève". En effet, la disparition de Mlle. Djeck vient s'ajouter à celle d'autres pachydermes l'ayant précédée - tels l'éléphant anonyme cité par F. Mayor tué à Genève en 1820 ou encore Chunee, mis à mort en 1826 -, mais aussi d'autres venant après elle comme Big Charlie, empoisonné en 1901.

12 La lithographie en question, intitulée Miss Djeck, 20 Avril 1837, réalisée par Dunant d'après un lavis de Rodolphe Töpffer, se trouve actuellement à la Bibliothèque de Genève. 
celebrity" (Hensbergen, 2018: 54). En effet, comme lors de ses représentations, la boucherie apparaît bien comme un nouvel espace dans lequel Djeck est exposée et mise à proximité du public-consommateur qui est ainsi attiré en foule. Face à la reproduction de l'image de la vedette dans des supports multiples comme moyen d'élargir et approfondire les rapports intimes avec celle-là, l'achat de la chair de Mlle. Djeck ouvre la possibilité d'accéder, non seulement à quelque chose qui l'évoque -les portraits et les lithographies, par exemple- ou qui lui appartenait -les objets que Hensbergen signale dans son étude-, mais à une partie réelle d'elle-même. Convertie en viande, réduite à sa seule corporalité, le public est désormais en mesure de créer des liens avec l'actrice éléphantine jusqu'alors insoupçonnés. L'artiste passe de ce fait d'être vue et frôlée physiquement durant la représentation à être complètement appréhendée, possédée par le public qui s'en approprie via l'achat et la consommation de sa chair. De leur côté, les connexions construites par les spectateurs à travers la presse cessent d'être virtuelles, l'ingestion de leur vedette sous forme de morceaux de viande leur permettant d'établir une relation d'intimité essentiellement physique qui, maintenant, est réelle et tangible. Finalement, il est intéressant de noter que la réduction totale de la vedette animale à l'état de bien de consommation est dans ce cas évidente et permet d'envisager le phénomène de la célébrité comme un mécanisme d'assujettissement et de réification, du moins lorsqu'il s'agit d'acteurs quadrupèdes, ce qui sera confirmé dans le versant publicitaire ultérieurement abordé.

Or, sa disparition est loin de supposer la fin de l'exposition de Mlle. Djeck qui demeure exhibée auprès d'un public qui peut désormais continuer de consommer une célébrité post mortem. Démembrée, la figure célèbre que Mlle. Djeck incarne est dédoublée et montrée simultanément dans le Musée d'histoire naturelle de Tournai (Belgique) et le Musée d'art et d'histoire de Genève (Suisse) qui conservent, aujourd'hui encore, sa peau et son crâne respectivement. Au-delà de l'intérêt scientifique, censé être l'objectif premier et principal de ces institutions qui se popularisent au cours du XIX ${ }^{\mathrm{e}}$ et début du XX $\mathrm{XX}^{\mathrm{e}}$ siècle, il existe ici une connexion permettant, sur le modèle du théâtre, de reconnaître dans le musée un nouvel espace d'exhibition de la vedette animale. À ce propos, Jane C. Desmond affirme que dans le contexte des expositions taxidermiques "the relationship between viewer and object is fundamentally theatrical" (2016: 33), arrivant même à les qualifier de "théâtres des morts". La recherche de réalisme est sur laquelle repose l'illusion de retour à la vie, une sorte de résurrection qui, replaçant Mlle. Djeck dans une scène différente, la réinscrit dans une logique spectaculaire. Ceci est d'autant plus évident que maintes fois les animaux sont présentés dans des dioramas qui ont pour but de reconstruire le contexte supposé naturel auquel ils appartiennent, l'animal rejoignant ainsi le rôle d'acteur. Cette mise en scène naturalisée et naturalisante est loin de répondre aux seuls critères taxonomiques et/ou d'ordre scientifique, le composant esthétique ou la capacité d'attraction étant également importants. Comme lors des spectacles zooscénographiques dans lesquels Djeck participait, les idées d'exotisme, 
d'inouï et d'attraction-magnétisme déterminent, et ce notamment au XIX ${ }^{\mathrm{e}}$ siècle, la sélection des spécimens exhibés, et par extension, les reconstructions dans lesquelles ils sont intégrés. En conséquence, les questions de la singularité ou différence et la curiosité du public, essentielles dans le phénomène du vedettariat, émergent aussi dans ces institutions comme éléments clés autour desquels s'articule l'exposition de l'animal mort. En ce sens, comme Peta Tait le souligne, "a visual perception of an animal fur skin in a life-like pose allows human curiosity to be satisfied, and in a close proximity [...] [and,] even though the posed animal body is completely immobile, it is not meant to be perceived by spectators as a dead body" (2015: 114). Dans le cas concret de Mlle. Djeck, la transposition analogique entre théâtre et musée suppose une diversification dans les espaces d'exhibition et permet le renouvellement des mécanismes d'accès à la figure animale. En effet, "the physical intimacy engenders emotional intimacy" (Poliquin, 2012: 136), l'exposition taxidermique rendant donc possible que les spectateurs continuent de satisfaire leur aspiration voyeuristique, et par là même, ce désir d'intimité envers la célébrité auparavant évoqués.

Finalement, le fait que la mort atteigne la sphère du public reste révélatrice, autant de l'ambivalence fondatrice du statut de célébrité ou vedette, que des particularités et divergences entre humains et animaux. En ce sens, bien que les exécutions publiques d'hommes et femmes continuent d'avoir lieu à l'époque ${ }^{13}$, tout comme celle de Djeck avant mentionnée, dans le contexte de l'humain, la mort, ainsi que les rituels et événements qui en dérivent, restent généralement du domaine du privé. L'exception étant certainement les funérailles des grandes personnalités, vedettes et artistes nationaux, qui prennent un caractère public, voire massif, tels les cas de Talma ${ }^{14}$ au XIX ${ }^{\mathrm{e}}$ siècle ou de Sarah Bernhardt au XX $\mathrm{XX}^{\mathrm{e}}$ siècle. Mais, ce qui reste essentiellement différent est le traitement des dépouilles. Ainsi, la possibilité de rendre public ce qui est en essence privé- à savoir, lesdites dépouilles -ne semble possible et entièrement acceptable, à quelques exceptions près, que lorsqu'il s'agit de restes animales (Desmond, 2016: 42-43). Si des exemples échappent à la norme, c'est parce qu'ils répondent à deux critères: la célébrité de la personne et/ou son identité animalisée, l'un des deux devant être nécessairement présent. Outre le cas cité par Jane C. Desmond (2016: 43) de Vladimir Lenin, qui remplit uniquement la première des conditions -donc la notoriété publique-, il est également possible de considérer les figures publiques de Saartije Baartman, plus connue comme la Vénus hottentote, ou de Julia Pastrana. En effet, même si ce fut involontairement, toutes deux participèrent de l'industrie du spectacle du fait de leur corporalité biopolitiquement non normative par rapport à une règle édifiée en accord avec le modèle occidental, devenu alors le paradigme

13 La dernière exécution publique en France a lieu le 17 juin 1939 devant la prison de Versailles, le condamné à mort étant Eugène Weidmann. Bien que la peine de mort ne soit abolie en France qu'en 1981, il est cependant important de noter que dès l'avènement de la III ${ }^{\mathrm{e}}$ République (1870) la question de la publicité des exécutions devient un enjeu de discussion, les autorités tâchant de plus en plus de les soustraire de la vue et la voie publiques.

14 Charles François Jean Baptiste Moreau (1827: 90) recense jusqu'à cent mille personnes lors des funérailles de François Talma; "une longue chaînes de voitures suivaient le corbillard, et plus de 80,000 personnes accompagnèrent son corps au tombeau" selon Augustus Pugin (1829: 150). 
de la correction corporelle (Ramos-Gay, 2015). Cet écart que leurs corps matérialisent résulte dans l'établissement d'analogies avec le règne animal auquel elles sont identifiées, constituant en même temps l'élément justificatif de leur exhibition publique. Parce qu'associées à une identité hybride qui les fait participer de l'animalité, ce que, tel que précédemment démontré, est accentué par le phénomène de la célébrité, l'exposition de leurs dépouilles ne semble contrevenir aucune limite éthique et/ou esthétique, qui autrement conduit obligatoirement à la désindividuation et anonymisation des restes humains exhibés (Desmond, 2016). L'identité animale à laquelle sont assimilées, unie à leur statut de vedettes du freak, rend donc possible la spectacularisation de leurs corps morts, alors même qu'elles maintiennent leur individualité, ce qui suppose, en dernière instance, l'exhibition et la publicitation du privé.

L'absence d'histoire serait précisément ce qui empêche la singularisation de l'animal vivant derrière les restes et ferait par extension acceptable son exposition publique (Poliquin, 2012; Desmond, 2016). Plutôt conçu comme représentatif ou modèle de l'espèce à laquelle il appartient, ce qui est également valable pour le cas des zoologiques, l'animal mort est dépourvu de la singularité que sa peau semblerait pourtant lui garantir. Cette structure d'anonymisation, comme le relèvent Rachel Poliquin (2012) et Jane C. Desmond (2016), est cependant inopérante lorsque le spécimen en question est pris au milieu des dynamiques du phénomène de la célébrité. Ainsi, le statut de vedette de Mlle. Djeck est également confirmé par le traitement différencié que l'on fait de l'exhibition taxidermique de ses dépouilles. D'une part, l'exploitation que le musée fait de son passé extraordinaire en tant que "star internationale au XIX ${ }^{\mathrm{e}}$ siècle", comme elle est encore qualifiée de nos jours ${ }^{15}$, permet de reconnaître le statut de célébrité qu'elle possédait de son vivant. Le recours à son histoire, résultat de son inclusion au sein de l'industrie spectaculaire du XIX ${ }^{\mathrm{e}}$ siècle, redonde de ce fait dans son individuation. Ce n'est donc pas la peau d'un éléphant asiatique quelconque que le musée exhibe, mais bel et bien les restes de Mlle. Djeck, actrice boulevardière occupant le premier rôle de L'Éléphant du roi de Siam. Or, la curiosité que Djeck suscitait chez les spectateurs dix-neuviémistes est transférée au public contemporain, l'intérêt qu'elle revêtait pour les premiers expliquant et déterminant, en dernière instance, l'attention qu'elle fait naître dans les seconds. Ceci d'autant plus que les possibilités de voir des spécimens animaux sont, de nos jours, beaucoup plus variées et accessibles qu'elles ne l'étaient à l'époque. Finalement, du fait de son caractère singulier, témoigné par les reportages et articles parus à la suite de son inclusion parmi les Trésors de la Fédération Wallonie-Bruxelles en 2017, Djeck s'avère être également une réclame publicitaire pour le musée, lui permettant d'attirer un plus large public. Elle retrouve, de ce fait, une valeur économique et publicitaire déjà exploitée par les salles, directeurs et propriétaires lors de sa carrière d'actrice pendant la première moitié du $\mathrm{XIX}^{\mathrm{e}}$ siècle.

15 Voir: Le fabuleux destin de Miss Djeck, l'éléphante du musée d'histoire naturelle de Tournai, article publié le 08 mars 2018 à < https://www.lavenir.net/cnt/dmf20180308_01136674/video-le-fabuleux-destin-de-miss-djeck-1elephante-du-musee-d-histoire-naturelle-de-tournai $>$ [21/04/2021]. 
D'autre part, la mise en scène muséale de l'actrice pachyderme à l'institution de Tournai, par opposition à la présentation décidée aux autres animaux, cherche à mettre en valeur son caractère exceptionnel. Après une longue salle où, à une partie et autre de la même, se succèdent grand nombre de spécimens différents - girafes, lions, zèbres, rhinocéros, chameaux, singes, etc. - qui se côtoient les uns les autres, l'on aboutit à une grande pièce au centre de laquelle est placée Djeck, seulement accompagnée d'un éléphant africain. Surélevée par une plateforme, Mlle. Djeck est (co)protagoniste d'un espace aux réminiscences théâtrales. Le fait de se trouver presque seule dans un scénario dépourvu d'autres accessoires que les seuls paneaux informatifs qui entourent les deux éléphants met en lumière et confirme le caractère unique de l'actrice pachyderme face aux autres animaux exhibés de manière conjointe. Cette disposition spatiale, unie à la récupération de son histoire, dotent la reconstruction taxidermique de Mlle. Djeck de toute une narrativité qui résulte de sa condition de vedette et lui assurent, encore aujourd'hui, le statut d'individu-animal. En conséquence, la superposition entre la situation de Saartje Baartman et de Juia Pastrana et celle de Mlle. Djeck est possible du fait de leur inclusion dans le circuit du spectacle. Ce dernier les fait accéder au statut de célébrités, phénomène par le biais duquel leurs identités respectives tendent à une mixité espéciste qui permet une exhibition post mortem individualisante dans la mesure où les premières sont rabaissées au rang d'animaux, l'éléphant(e) retrouvant, de son côté, un espace de reconnaissance principalement réservé aux acteurs humains.

\section{Promotion, publicité et construction de l'image publique de la célébrité animale}

Parallèlement, et outre la question de l'intimité qui vient d'être analysée, les articles et écrits publiés sur Mlle. Djeck,

Prétendent répondre à un appel du public et à une nécessité sociale, de fait, ils se conforment à une exigence médiatique, celle de la réclame, et ce faisant, ils contribuent à structurer un imaginaire collectif autour de la notion de vedette ou d'étoile. (Thérenty, 2013: s/n).

Autrement dit, la presse, mais aussi les mémoires, biographies et autres formes de médiatisation qui se trouvent au cœur des dynamiques participant de la reconfiguration des espaces public et privé, puis des rapports public-vedette, deviennent simultanément instruments publicitaires à l'origine et au service du phénomène de la célébrité. En témoignent l'exemple du combat tenu en 1831 à Liverpool contre la lionne Fanny dans lequel Mlle. Djeck semble avoir sauvé la vie de son propriétaire $M$. Huguet, ce que les journaux interprètent de manière généralisée comme une démonstration de l'attachement que l'animal éprouve pour son propriétaire $^{16}$. À cet effet, le Manuel de littérature française à l'usage des allemands de Joseph

16 Voir les journaux: Le Corsaire du 3 avril 1831; La Gazette des théâtres du 18 mars 1832. Voir: Le Cirque 
Gischig, consacre une entrée à ce combat dans laquelle il confirme que "le bruit de cette prouesse attira le lendemain au Cirque un concours extraordinaire de spectateurs, empressés d'admirer l'intelligent et courageux animal [...]" (1853: 59). Ceci prouve que, de son vivant, la diffusion de ces anecdotes agit également en moyen de promotion de la figure célèbre que Mlle. Djeck représente. La valeur publicitaire de ces écrits permet alors d'accroître la popularité de l'artiste qui s'affirme, à son tour, en principal mécanisme de réclame pour les différents théâtres et salles de représentation.

Cependant, ce sont les attaques dont l'actrice éléphantine est protagoniste, et qui laissent des blessés de diverse considération, parfois même des morts, celles qui mettent le mieux en relief l'importance de la presse dans les logiques commerciales et de propagande propres au dispositif de la célébrité17, ainsi que dans la construction de l'image (publique) de la vedette. Souvent justifiée par les journalistes qui s'en font écho, Djeck est déchargée d'une responsabilité généralement attribuée à l'inconscience et/ou malveillance des humains. Deux conclusions peuvent donc être tirées vis-à-vis de cette attitude de la part des journalistes. La première des options est qu'il s'agisse d'une vraie campagne publicitaire orchestrée par ses propriétaires et les directeurs des salles dans lesquelles elle se produisait. Ainsi, si les rumeurs et scandales de la vie privée ont le pouvoir d'accroître l'intérêt, en l'occurrence personnel, pour un(e) artiste, engendrant cette illusion d'intimité avant évoquée et agissant en éléments potentiels de publicité; l'agressivité de Mlle. Djeck-dans le cas de se montrer récurrente et systématique - peut, au contraire, s'avérer un agent de dissuasion. Se retrouver dans la même salle qu'un animal qui possède une certaine liberté de mouvements et un caractère colérique peut être vu par les spectateurs comme étant trop dangereux pour assumer le risque d'assister à ses représentations. Ceci d'autant plus que, vers la décennie de 1830, d'autres éléphants, notamment Kiouny, se produisent parallèlement dans les théâtres parisiens, mais aussi en province. Cette mauvaise presse nuirait donc, non seulement l'image et la carrière de Mlle. Djeck, mais surtout les bénéfices que ses propriétaires et les directeurs de théâtre tirent des productions dans lesquelles elle participe.

Cette position est précisément celle que Charles Reade propose dans la nouvelle intitulée Jack of all Trades. A Matter of Fact Romance qu'il publie en 1858 dans le Harper's Magazine. Dans cette œuvre fictionnelle fondée sur des faits réels, Reade, à travers le personnage de Lott, soutient que la prédisposition de l'éléphant(e) à la violence est cachée délibérément par ses successifs cornacs. D’après la dénonciation de Lott-Reade, tous les écrits consommés

Olympique volume anonyme de 1832 imprimé à Poitiers. Voir aussi: de Carnerero, José María. 1832. Cartas Españolas ó sea Revista semanal, histórica, científica, teatral, artística, crítica y literaria. Madrid, Imprenta de I. Sancha, Tomo IV, p.56.

17 Les attaques continuent d'être citées de nos jours dans les moyens de communication qui traitent de la reconstruction et nouvelle exhibition des dépouilles de Mlle. Djeck dans le musée belge de Tournai. Ainsi, le caractère violent, uni à son passé d'actrice célèbre, agissent encore aujourd'hui en tant qu'agents publicitaires visant à attirer le public, puis à augmenter les visites, voire bénéfices économiques de l'institution, pour qui l'éléphant(e) s'avère une de ses réclames. 
par le public, dans la ligne de ceux qui ont été antérieurement recensés, résultent du processus par lequel est créée "[the] image management" (Tolson, 2001), c'est-à-dire, l'image publique de la vedette ou $\operatorname{star}^{18}$. Les articles parus après l'exécution de Mlle. Djeck qui ont pour objectif de justifier cette décision polémique, unis aux références (certes exiguës) publiées de son vivant à propos de ces événements violents, invitent à présumer la véracité de leur existence. L'intérêt de Jack of all Trades réside, d'un côté, dans le fait que Charles Reade met en lumière la place centrale de la presse dans le surgissement et consolidation de la figure de la vedette. D'un autre côté, l'écrit souligne l'importance du composant commercial, de même que la question de l'imposture dans le phénomène de la célébrité. En ce sens, l'auteur met l'accent sur la construction de l'actrice éléphantine comme un "phénomène médiatique" (Blewitt, 2013: 329), comme étant essentiellement le résultat de logiques publicitaires et de consommation qui articulent le monde du spectacle. En effet, ceci constitue une des principales critiques traditionnellement érigées contre la figure de la vedette, voire de la culture de la célébrité, ainsi réduites à simple stratégie de promotion (Filippi, Harvey \& Marchand, 2017) et "objet de propagande" (Percheron, 2016: 90) respectivement.

Notons, toutefois, que la responsabilité est attribuée, non pas au pachyderme, mais aux humains (propriétaires, cornacs, directeurs de théâtre, etc.) qui font ici l'office de manager. En conséquence, le phénomène de la célébrité ne peut être lu pour les animauxacteurs en termes d'émancipation, mais, au contraire, de soumission-réification, bien que paradoxalement le vedettariat conduise à une individuation de l'animal s'éloignant, comme il a été déjà vu, de l'anonymat et de sa conceptualisation générique à l'intérieur de la diluante catégorie d'espèce. De ce fait, à la différence de vedettes humaines comme Sarah Bernhardt qui font un usage conscient et délibéré de la presse comme moyen publicitaire qu'elles contrôlent personnellement (Ramos-Gay, 2018: 65) -ce qui leur permet de retrouver le statut de leurs congénères masculins-, dans le cas des vedettes animales "this expansion across many media enhanced the value of Jumbo, [Mlle. Djeck] and similar celebrities as commodities" (Blewitt, 2013: 329). Cette conversion de la vedette animale en marchandise consommable est totale lorsque l'animalia se transforme explicitement en objet, trait ce dernier qu'elle partage avec les célébrités humaines. Les lithographies ${ }^{19}$,

18 Bien que Mlle. Djeck ne soit pas explicitement justifiée dans le récit de Charles Reade, la description de scènes qui relèvent les instruments de torture, notamment la pique, utilisés lors de son dressage, mais surtout la réaction de terreur de l'éléphant(e) face à cet outil, invitent le lecteur à éprouver un sentiment de compassion et d'empathie envers l'animal. En quelque sorte, le fait de dévoiler le caractère systématique de ces pratiques cruelles bénéficie l'image de la pachyderme car, tout en acceptant la véracité de son caractère violent, ce dernier peut apparaître comme conséquence directe de son existence tragique. Mlle. Djeck apparaît ainsi comme victime d'un système physique (le dressage) et symbolique-économique (l'impérialisme, mais aussi le composant capitaliste qui régit une partie de la culture de la célébrité) d'oppression qui favorise la durabilité de son image publique essentiellement positive.

19 Pour voir la lithographie représentant Mlle. Djeck à côté d'un squelette de baleine, certainement renvoyant à l'idée de la taille comme élément singularisant et différenciateur, aller sur: <https://gallica.bnf.fr/ark:/12148/ btv1b8437440z.r=djeck?rk=21459;2>[12/03/2021]. 
assiettes en porcelaine ${ }^{20}$ ou volumes ${ }^{21}$ décorés avec ou présentant Mlle. Djeck la convertissent en matière -dans le sens étymologique du terme- prête à être consommée, situation, nous l'avons vu, qui trouve son expression la plus extrême lorsque l'éléphant(e) per se, et non pas son image, est vendue comme bien alimentaire et de consommation.

La deuxième option qui explique l'acquittement de Mlle. Djeck de la part de la presse est que celui-ci réponde à une image essentiellement positive que l'éléphant(e) s'était forgé(e) auprès du public. En ce sens, l'actrice jouirait de l'affection de la part des spectateurs, un attachement que les journaux reproduiraient et alimenteraient à leur tour. En témoigne l'adaptation française, publiée dans la Revue Britannique, de la nouvelle Jack of all Trades. A Matter of Fact Romance (1858). Cette version reste remarquable du fait du parti pris en faveur de l'artiste dont l'intégrité est défendue, autant implicitement que par la note finale qui clôt la narration. Cette note, qui cherche à s'éloigner ouvertement de l'original “de peur d'attribuer des crimes imaginaires à mademoiselle Djeck" (Pichot, 1858: 223), sert d'introduction à une anecdote qui vise à la "réparation des crimes injustement attribués sans doute à mademoiselle Djeck, sinon à Kalisch" (223). David C. Giles explique cette persistance de la sympathie envers l'animal parce que "[the] animals celebrities are invested with affective power, [and because] this power can never be compromised by accusations of emotional manipulation" (Giles, 2013: 116). Ce que l'auteur suggère c'est que la question de l'imposture n'opère pas lorsqu'il s'agit de vedettes animales, la dénonciation de manipulation en raison de stratégies de publicité et de logiques économiques étant uniquement imputables à l'humain. C'est ce que Rachel Poquilin nomme "the intrinsic material fact of animal authenticity" (2015: 93) et la conduit à affirmer, aussi pour le contexte des exhibitions taxidermiques avant analysées, que "taxidermied beasts [...] materialized 'truths' by being authentic animal skins" (95). Ceci permet de retrouver le récit premier de Charles Reade qui dans sa nouvelle fait retomber la responsabilité d'une image publique qui rompt avec la notion d'authenticité, non pas sur Mlle. Djeck, mais sur les directeurs de théâtre et/ou ses cornacs. Cette situation expliquerait donc l'estime générale que la presse et l'opinion publique ressentent pour l'éléphant(e) qui se traduit dans la promotion et diffusion d'une image éminemment positive. La défense que ces écrits font de Mlle. Djeck met en lumière le rôle de la presse comme intermédiaire indispensable dans la construction de l'image publique de la vedette, ce qui n'exclue pourtant pas le fait qu'elle agisse en outil médiatique de propagande. L'omniprésence de Mlle. Djeck dans les journaux, quotidiens, voire dans le genre littéraire et fictionnel, durant sa vie, mais aussi après sa mort, vient s'ajouter en tant que preuve de sa

20 Entre 2017 et 2018, des assiettes reproduisant Mlle. Djeck dans l'une des scènes du spectacle l'Éléphant du roi de Siam ont été vendues aux enchères à Carmarthen Antiques \& Flea Market, au Royaume Uni.

21 En 1829 paraît sous le titre de A peep at the Elephant at the Adelphi Theatre, un volume composé de quatre planches contenant des images colorées sur les représentations de Mlle. Djeck au théâtre londonien Adelphi. Le livret se trouve actuellement à la National Art Library qui dans la description affirme que la popularité de la pièce expliquerait que le volume fût probablement vendu sur place comme souvenir. Pour accéder aux informations et images voir: $<$ https://collections.vam.ac.uk/item/O1387123/a-peep-at-the-elephant-paper-peepshow/> [12/03/2021]. 
pleine participation dans le système de vedettariat et confirme définitivement son statut de célébrité-vedette au sein des arts du spectacle de la première moitié du XIX siècle.

\section{Conclusion}

En accord avec ce qui vient d'être exposé, il est possible de constater l'application des mêmes paradigmes pour le vedettariat humain et animal, ceci grâce, en premier lieu, à l'identité éminemment hybride qui vient caractériser la vedette animale. Ce caractère liminaire est celui qui permet sa pleine intégration dans les mécanismes et stratégies liés au phénomène de la célébrité, bien qu'elles aient été essentiellement développées pour analyser des exemples d'acteurs et actrices humains. Ainsi, l'identité animale, voire l'animalité première de Djeck, devient ce It analysé par Joseph Roach (2005), et déjà souligné par Elinor Glyn en 1927 pour les félins, cet élément sine qua non pour accéder à la célébrité et qui a été aussi défini comme magnétisme, attraction ou aura. Au simple statut d'animal vient s'ajouter la curiosité que l'actrice éléphantine suscite du fait de sa taille, de sa provenance extraeuropéenne, donnant de ce fait corps aux idées de rareté et de différence qui redondent dans un magnétisme et une singularisation toujours croissants. Une fois immergée dans le circuit théâtral, c'est alors que le phénomène de la célébrité agit en agent déstabilisateur anthropomorphisant Mlle. Djeck qui accède ainsi au statut d'animal humanisé, et par là même, à celui de "elephantine star". Cette humanisation, performative autant que discursive, conduit à une identification partielle avec l'humain qui résulte de et dans son inclusion au système de vedettariat. La célébrité est ainsi capable de dissoudre les différences entre espèces et de faire converger dans un même individu, humain ou animal, des caractéristiques qui résultent dans une identité à mixité espéciste. Parallèlement, l'étude met en lumière que la célébrité animale est aussi un phénomène médiatique dépendant de logiques commerciales et publicitaires qui déterminent aussi le succès de la vedette, et par conséquent, de ses propriétaires, puis des directeurs de salles théâtrales. Ce composant économique met en lumière le danger de la réification de la figure célèbre qui frôle alors le statut d'objet d'exhibition et de consommation, situation emblématiquement exemplifiée par la transformation de Djeck en viande. Basculant entre l'animal et l'humain, puis entre le statut d'individu, voire sujet, et celui d'objet, la célébrité émerge bien comme phénomène d'ambivalence ontologique.

Dans le même temps, le vedettariat contribue à la singularisation de l'artiste auprès des autres acteurs, qu'ils soient bipèdes ou quadrupèdes, avec lesquels Mlle. Djeck partage la scène et confirme sa position de protagoniste. Désormais, la vedette capitalise l'intérêt des spectateurs, voire de l'opinion publique, et se substitue aux rôles qu'elle avait jusqu'alors incarnés. C'est ce que met en lumière la progressive disparition d'articles centrés sur la critique théâtrale du spectacle de L'Éléphant du roi de Siam en faveur de la multitude de références journalistiques consacrées à l'animalia de Mlle. Djeck. La célébrité devient donc un mécanisme privilégié 
d'individuation, capable même de rompre avec la limitation temporelle que la mort impose. Tirée de l'anonymat, et par conséquent de l'oubli, son statut de vedette explique donc sa présence dans les journaux, le genre fictionnel, les anecdotes littéraires ou dans les musées-théâtres des morts, cela même après son exécution à Genève. Sans la popularité conquise de son vivant, les dépouilles de Mlle. Djeck n'auraient peut-être jamais été acceptées par les musées de Tournai et de Genève. Mais, ce qui reste certain, c'est qu'elles auraient été présentées parmi les innumérables dioramas comme simples modèles ou types représentatifs de l'espèce, dépourvues de toute narrativité, donc de l'histoire et des coordonnées qui l'inscrivent dans un temps et un lieu concret, garants de la perdurabilité de son identité. Ici réside une des principales particularités venant distinguer les vedettes humaines et animales. D'une part, l'atemporalité de son exhibition, ce qui entraine la diversification des espaces de consommation de la célébrité, les lieux nouveaux d'exposition et approximation de la figure célèbre se constituant, par effet d'analogie, sur le modèle du théâtre. D'autre part, la transgression, voire suppression totale des frontières entre ce qui reste du domaine public et privé que les expositions taxidermiques, ainsi que les moyens de médiatisation comme la presse, exemplifient.

En effet, les nouvelles sur les événements personnels les plus banals, la prolifération de rumeurs (macabres, polémiques ou controversés) ou les enterrements publics mettent en lumière cette redéfinition des événements appartenant à l'ordre du privé, élément celui-ci caractéristique de la célébrité. C'est ce que prouve la reproduction du lever de Voltaire sous forme de peinture signée Jean Hubert (circa 1772), la multitude présente aux funérailles de Talma, la répercussion dans la presse des scandales dont Lord Byron fut protagoniste, mais aussi les rumeurs surgies sur les liaisons amoureuses de Mlle. Djeck ou le naufrage dans lequel elle aurait péri, son exécution publique ou son exhibition post mortem ultérieure. Ainsi, l'attrait croissant pour un artiste concret se traduit dans un désir d'intimité que les spectateurs tentent de combler par une recherche de proximité, autant physique que virtuelle. Les planches fournissent un premier milieu de rapprochement qui alimente le penchant voyeuriste du spectateur-admirateur, mais elles restent toujours un lieu de fictionnalité, voire d'imposture, qui ne satisfait qu'une partie de ses atteintes. En effet, tout en étant plus proche des concepts de réel et d'authenticité que son identité animale lui confère, sur la scène Mlle. Djeck représente un personnage qui est autre qu'elle-même, vrai objet d'intérêt du public. Entrant dans l'intimité et la vie privée, la presse est en mesure de créer une illusion de familiarité avec la pachyderme de sorte que le public renforce le sentiment d'une proximité virtuelle ou à distance. À cet égard, les expositions de Mlle. Djeck fondées sur sa corporalité -boucherie et exhibitions muséales- transforment la virtualité des rapports entretenus au travers des différents moyens de médiatisations écrits, ou par l'image, en une connexion réelle, physique et matérielle. Ainsi, bien que traditionnellement considéré dès une perspective anthropocentrique, l'analyse des dynamiques sus-citées et ayant participé à la constitution de Mlle. Djeck en bête de scène, montre bien que le phénomène de la célébrité peut être également appliqué au contexte des animaux-acteurs. 
Anales de Filología Francesa, n. ${ }^{\circ}$ 29, 2021

$\mathrm{M}^{\mathrm{a}}$ TERESA LAJOINIE DOMÍNGUEZ

\section{Références bibliographiques}

Actors by Daylight or Pencilings in the Pit. 1838. Londres, J. Pattie, vol. I.

Anonyme. 1832. Le Cirque Olympique. Poitiers. <https://gallica.bnf.fr/ark:/12148/bpt6k9615282n.r=le\%20cirque\%20olympique\%201832?rk=21459;2> [05/05/2021].

BLEwitT, John. 2013. "What's new pussycat? A genealogy of animal celebrity" in Celebrity Studies, $\mathrm{n}^{\mathrm{o}} 4$ (3), 325-338.

Brown, Thomas. 1834. Anecdotes of the Animal Kingdom; containing Illustrations of the Caracters, Habits, Dispositions, and Capabilities of Quadrupeds, Bipeds, Reptiles, and Insects. Glasgow, Archibald Fullarton \& Co.

Burney, Charles. 1773. The Present State of Music in France and Italy. London, Printed for T. Becket and Co. Strand, 2ème éd.

CANSELIER, Sonia. 2017. "Les grands progrès de la protection animale en droit français et européen" in Histoire de la recherche contemporaine, tome IV, $\mathrm{n}^{\circ} 1:<\mathrm{http}$ ://journals.openedition.org/hrc/977> [25/03/2021].

Danahay, Martin A. 2007. "Nature Red in Hoof and Paw: Domestic Animals and Violence in Victorian Art" in Morse, Denenholz Deborah \& Martin A. Danahay (éds.). Victorian Animal Dreams. Representations of Animals in Victorian Literature and Culture. Aldershot, Ashgate Publishing.

Descola, Philippe. 2005. Part-delà la nature et culture. Paris, Gallimard.

Desmond, Jane C. 2016. Displaying Death and Animating Life: Human-Animal Relations in Art, Science, and Everyday Life. Chicago, The University of Chicago Press.

Dubors, Vincent. 2001. Legitimation CNRS. Dictionnaire des politiques culturelles de la France depuis 1959. Paris, Larousse, 366-368.

Dupas, Fanny. 2005. Le statut juridique de l'animal en France et dans les états membres de l'Union Européenne: historique, bases juridiques actuelles et conséquences pratiques. Thèse Docteur en Vétérinaire, Université Paul-Sabatier.

Fennell, James. 1841. A Natural History of British and Foreing Quadrupeds; containing many Modern Discoveries, Original Observations and Numerous Anecdotes. London, Joseph Thomas, Finch Lane, Cornhill.

FilipPI, Florence, Harvey Sara \& Sophie Marchand (sous la direction de). 2017. Le sacre de l'acteur. Émergence du vedettariat théâtral de Molière à Sarah Bernhardt. Paris, Armand Colin.

GaUTIER, Théophile. 1859. Histoire de l'art dramatique en France depuis vingt-cinq ans. Paris, Éditions Heztel, 4 série.

Geneviève, Faye. 2010. "Le renouvellement des salles théâtrales à Paris après le décret de 1864" in Yon, Jean-Claude (dir.). Les spectacles sous le Second Empire. Paris, Armand Colin, 61-71.

GILES, David C. 2013. “Animal celebrities" in Celebrity Studies, nº 4 (2), 115-128. 
Anales de Filología Francesa, n. ${ }^{\circ}$ 29, 2021

DJECK OU LA STARIFICATION ANIMALE DANS LA PREMIÈRE MOITIÉ DU XIXE SIÈCLE

Gischig, Joseph. 1853. Manuel de littérature française à l'usage des allemands. Vienne, Charles Gerold et Fils, Libraires-Éditeurs.

Goldsmith, Oliver. 1842. History of Man and Quadrupeds. London, Andrew Moffat, Vol. II.

Gouabault, Emmanuel \& Claudine Burton-Jeangros. 2010. "L'ambivalence des relations humain-animal. Une analyse socio-anthropologique du monde contemporain" in Sociologie et sociétés, $\mathrm{n}^{\circ}$ XLII (1), 299-324.

Hughes, Joseph (édité par). 1883. The Practical Teacher: A Monthly Educational Journal. London, Joseph Hughes, Pilgrim Street, Ludgate Hill, Vol. II.

Jones, Emrys D. \& Victoria Joule (édité par). 2018. Intimacy and Celebrity in EighteenthCentury Literary Culture. Public Interiors. London, Palgrave Macmillan.

Karr, Alphonse. 1835. "Spectacles de Paris" in Le Monde Dramatique. Revue des spectacles anciens et modernes, vol. 1, deuxième édition, 167.

Koenigsberger, Kurt. 2007. The Novel and the Menagerie. Totality, Englishness, and Empire. Columbus, The Ohio State University Press.

Le Charivari, lundi 10 juillet 1837, sixième année, numéro 191.

Le Corsaire, mardi 18 janvier 1831, IX année, numéro 2911.

Le Figaro, dimanche 5 février 1832, VII année, numéro 36.

Le Journal des comédiens, dimanche 5 juillet 1829, première année, numéro 28.

Le Journal des débats politiques et littéraires, jeudi 6 juillet 1837.

Le Mercure de France au dix-neuvième siècle. 1829. Paris, Au Bureau du Mercure, Tome vingt-sixième.

Le Messager des chambres, mercredi 9 décembre 1829, numéro 343.

Le Messagers des chambres, lundi 6 juillet 1829, numéro 187.

LiLTi, Antoine. 2014. Figures publiques. L'invention de la célébrité (1750-1850). Paris, Fayard (coll. L'épreuve de l'histoire).

Losano, Antonia. 2017. "Performing Animals/Performing Humanity" in Mazzeno, L. \& R. Morrison (éds.). Animals in Victorian Literature and Culture. London, Palgrave Macmillan, 129-146.

Lukhurst, Mary \& Jane Moody (édité par). 2005. Theatre and Celebrity in Britain 16602000. Basingstoke, Palgrave Macmillan.

Miller, John. 2012. Empire and the Animal Body: Violence, Identity and Ecology in Victorian Adventure Fiction. London, Athem Press.

Moreau, Charles François Jean Baptiste. 1827. Mémoires historiques et littéraires sur F.-J. Talma. Paris, Chez l'Advocat Libraire, troisième édition, vol. 1-2. 
Morin, Edgar. 1957. Les Stars. Paris, Éditions du Seuil.

Percheron, Bénédicte. 2016. "Les hommes face aux animaux à Rouen au XIXe siècle Représentations, études zoologiques et perceptions" in Société française d'histoire urbaine $\mathrm{n}^{\circ} 47$ (3), 87-105.

Picнот, Amédée (sous la direction de). 1858. Revue Britannique. Recueil international. Bruxelles, Au Bureau de la Revue Britannique, tome troisième (édition franco-belge-nouvelle série).

Poliquin, Rachel. 2012. The Breathless Zoo: Taxidermy and the Culture of Longing. Pennsylvania, The Pennsylvania State University Press.

Pugin, Augustus \& Charles Heathe. 1829. Paris and its Environs. Displayed in a series of Picturesques views. London, Robert Jennings, vol. 1.

Ramos GAY, Ignacio. 2015. "El actor animalizado en las culturas parateatrales victorianas" in Navarro, A. \& J.J. Pomer (éd.). Bestiaris i metamorfosis a les literatures clàssiques $i$ la seua tradició. Amsterdam, Adolf M. Hakkert Publisher, 175-187 (Classical and Byzantine monographs édité par Giangrande, G. \& H. White).

Ramos Gay, Ignacio. 2018. “ 'Partly American!': Sarah Bernhardt’s Transnational Disability in the American Press (1915-1918)" in ATLANTIS Journal of the Spanish Association of Anglo-American Studies, $\mathrm{n}^{\circ}$ 40.2, 63-80.

RoACH, Joseph. 2005. "Public Intimacy: The Prior History of 'It"” in Lukhurst, Mary \& Jane Moody (édité par). Theatre and Celebrity in Britain 1660-2000. Basingstoke, Palgrave Macmillan, 15-30.

TAIT, Peta. 2015. "Fleshing Dead Animals. Sensory Body Phenomenology in Performance" in Bleeker, Maaike, Sherman, Jon F. \& Eirini Nedelkopoulou (édité par). Performance and Phenomenology. Traditions and Transformations. New York-Londres, Routledge, 111-120.

The Calcutta Magazine and Monthly Register, février 1830.

The Edinburgh Literary Journal; or, Weekly Register of Criticism and Belles Lettres. Stanford Library, June 1829 - December 1829, Edinburgh, Constable and Co., 19, Waterloo Place, 403.

ThÉREnTy, Marie-Ève. 2013. “Le récit de vie de vedette, L'invention d'un genre: Rigolboche, Thérésa, Paulus" in Belphégor [consulté le 05 mai 2019] <http:// journals.openedition.org/ belphegor/279>

Tillyard, Stella. 2005. "Celebrity in Eighteenth-Century London” in History Today, n' 55 (6), 20-27.

Tolson, Andrew. 2001. “'Being Yourself': The Pursuit of Authentic Celebrity” in Discourse Studies, $\mathrm{n}^{\circ} 3$ (4), 443-457.

Van Hensbergen, Claudine. 2018. “Anne Olfield's Domestic Interiors: Auctions, Material Culture and Celebrity" in Jones, Emrys D. \& Victoria Joule (édité par). Intimacy and Celebrity in Eighteenth-Century Literary Culture. Public Interiors. London, Palgrave Macmillan, $35-58$. 
